\title{
Measuring activity limitation outcomes in youth with spinal cord injury
}

\author{
MD Slavin ${ }^{1}$, MJ Mulcahey ${ }^{2,3}$, C Calhoun Thielen ${ }^{2}, \mathrm{P} \mathrm{Ni}^{1}$, LC Vogel ${ }^{4}, \mathrm{SM} \mathrm{Haley}^{1,5}$ and AM Jette ${ }^{1}$
}

Study design: A cross-sectional study.

Objectives: The Pediatric Spinal Cord Injury Activity Measure (PEDI-SCI AM), which includes calibrated item banks (child and parent versions) for general mobility, daily routines, wheeled mobility and ambulation, can be administered using computerized adaptive tests (CATs) or short forms (SFs). The study objectives are as follows: (1) to examine the psychometric properties of the PEDI-SCI AM item banks and 10-item CATs; and (2) to develop and evaluate the psychometric properties of PEDI-SCI AM SFs.

Setting: US Shriners Hospitals for Children (California, Illinois and Pennsylvania).

Methods: Calibration data from a convenience sample of 381 children and adolescents with SCI and 322 parents or caregivers were used to examine PEDI-SCI AM item banks, 10-item CATs and SF scores. We calculated group reliability, internal consistency (Cronbach's alpha) and interclass coefficients (ICCs) to assess agreement between 10-item CATs, SFs and item banks. The percent of the sample with highest (ceiling) and lowest (floor) scores was also determined. An expert panel selected items for 14 SFs.

Results: PEDI-SCI item banks, 10-item CATs and SFs demonstrate acceptable group reliability (0.73-0.96) and internal consistency (0.77-0.98). ICC values show strong agreement with item banks for 10-item CATs (0.72-0.99) and SFs. Floor effects are minimal $(<15 \%)$. Ceiling effects are minimal for children with tetraplegia but high in children with paraplegia for general mobility (13.41-26.05\%) and daily activities (12.99-32.71\%).

Conclusions: The PEDI-SCI AM exhibited strong psychometric properties for children with tetraplegia. Replenishment of the general mobility and daily routine item banks is needed to reduce ceiling effects noted for youth with paraplegia.

Spinal Cord (2016) 54, 546-552; doi:10.1038/sc.2015.194; published online 17 November 2015

\section{INTRODUCTION}

Relevant, precise and efficient measures are needed to examine the efficacy of rehabilitation programs for youth with spinal cord injury (SCI) and to support evidence-based practice. Generic measures currently used in this population have significant conceptual and psychometric limitations. ${ }^{1}$ For example, the PedsQL ${ }^{2}$ includes items inappropriate for children with SCI (for example, 'it's hard for me to walk more than one block' and 'it's hard for me to run'), and the WeeFIM may not detect clinically meaningful change. ${ }^{3}$ The Pediatric Spinal Cord Injury Activity Measure (PEDI-SCI AM) was developed specifically to assess activity outcomes in youth with $\mathrm{SCI},{ }^{2-5}$ providing an alternative to generic pediatric outcome measures. The PEDI-SCI $\mathrm{AM}^{4-8}$ includes activities important to youth with $\mathrm{SCI}$, and items assess a wide range of abilities in the following domains: general mobility, daily routines, wheeled mobility and ambulation.

Previous work determined item calibrations and verified PEDI-SCI AM item bank unidimensionality. ${ }^{8}$ PEDI-SCI AM calibrated item banks can be administered as computerized adaptive tests (CATs), which use a computer program to select items. A CAT begins with a mid-range item, and subsequent items are administered based on the individual's responses. Items that are too hard, too easy or irrelevant are not administered. ${ }^{9}$ As each item is administered, the score estimate precision increases. The program terminates, based on pre-determined rules specifying a level of precision (s.e.) or maximum number of items, to yield a final score estimate. Scores for a given domain can be compared on repeated measures even though different items are administered because scores are based on the same metric. Although there are many benefits to using CAT administration, static short form (SFs), comprising items carefully selected from calibrated item banks, are used when computer administration is not feasible.

The objectives of this study are to (1) examine psychometric properties of PEDI-SCI AM item banks and 10-item CATs) and (2) develop PEDI-SCI AM SFs and evaluate their psychometric properties.

\section{MATERIALS AND METHODS}

\section{Participant-calibration sample}

PEDI-SCI AM calibration data were obtained from a convenience sample of 381 youth with acquired SCI (traumatic, transverse myelitis, tumor and so on) for the child-reported version (age 8-21 years) and 322 parents/caregivers of children with SCI for the parent-reported version (age 4-21 years). Inclusion criteria were discharge from initial SCI rehabilitation with return to pre-injury environment for at least 3 months. As the measure was developed specifically for children with acquired SCI, children with congenital (for example, spina bifida) and progressive conditions (for example, spinal muscle atrophy) were excluded from the study. Other exclusion criteria were as follows: English not primary language; and brain injury that interfered with the ability to read, comprehend and respond to items. Data were collected within the Shriners

${ }^{1}$ Health and Disability Research Institute, Boston University School of Public Health, Boston, MA, USA; ${ }^{2}$ Department of Occupational Therapy, Jefferson College of Health Professions, Thomas Jefferson University, Philadelphia, PA, USA; ${ }^{3}$ Shriners Hospitals for Children, Philadelphia, PA, USA and ${ }^{4}$ Shriners Hospitals for Children, Chicago, IL, USA ${ }^{5}$ Deceased.

Correspondence: Dr MD Slavin, Health and Disability Research Institute, Boston University School of Public Health, 715 Albany Street, Talbot 5 West, Boston, MA 021118, USA. E-mail: mslavin@bu.edu

Received 21 April 2015; revised 2 September 2015; accepted 24 September 2015; published online 17 November 2015 
Hospitals for Children System (Philadelphia and Chicago, and Northern California hospitals). The study was approved by the Institutional Review Board of each facility. Parents and children provided consent and assent, respectively. We certify that all applicable institutional and government regulations concerning the ethical use of human volunteers were followed during the course of this research.

\section{PEDI-SCI CAT development and simulation}

The CAT algorithms were developed at Boston University for each domain and programmed to administer the first item with highest Fisher Information value at the average sample score level. Post hoc simulations were conducted on the calibration data set to estimate the 10-item CAT scores and s.e. The person score and s.e. were estimated using the weighted likelihood estimation method. ${ }^{10}$ Each subsequent item was selected based on maximization item Fisher information value at the current score level, and the score and s.e. were recalculated after feeding the program with known response until 10 items had been administered. The final scores were transformed to the $T$-score scale, with a population mean and s.d. of 50 and 10, respectively. Higher scores represented higher function.

\section{PEDI-SCI SF development}

An expert panel comprising 12 professionals ( 1 medical doctor, 1 psychologist, 4 physical therapists and 6 occupational therapists) attended a 1-day meeting to select SF item candidates from PEDI-SCI AM item banks by reviewing two item parameters: item difficulty (measured in logits) and item discrimination (measured as the slope of the item characteristic curve). Groups identified SF item candidates using spreadsheets with PEDI-SCI items hierarchically organized based on logit scores with an additional column for item characteristic curve slopes. Logit scores were used to select items with an appropriate range of difficulty, and item characteristic curve slopes identified items that best discriminated among persons with different ability levels. Finally, SF item candidates were reviewed from a clinical perspective to ensure that important aspects of function were included in each SF. Groups presented initial recommendations and used an iterative process to identify 7-12 items for the four domains, with separate SFs for child/parent respondents (eight initial $\mathrm{SFs})$. On the basis of the differences in abilities of youth with paraplegia and tetraplegia and experience developing the adult SCI-functional index (SCI-FI) SFs, ${ }^{11}$ separate paraplegia and tetraplegia SFs were developed for the daily routines and wheeled mobility domains (four additional SFs: two parents/ two children). Bowel and bladder management is critical for youth with SCI, and items assessing these abilities are in the item bank but were not selected for inclusion in the SF because the items did not have a high information function. Also, these items are not relevant for youth with SCI not using bowel and bladder programs and could not be scored. Separate SFs were developed for manual and powered wheeled mobility for youth with tetraplegia to yield a total of 14 separate PEDI-SCI SFs. Iterative psychometric analyses to examine properties of the $14 \mathrm{SFs}$ were conducted by two investigators (MDS and MJM) to ensure optimal item selection.

\section{Psychometric analyses}

Calibration sample data were used to calculate group-level reliability for the full-item banks, 10-item CATs and SFs for each domain (separate for child and

Table 1 Descriptive statistics of the PEDI-SCI parent-reported and child-reported general mobility and ambulation scales ${ }^{\mathrm{a}}$

\begin{tabular}{|c|c|c|c|c|c|c|c|c|}
\hline Respondent & Version & $\mathrm{N}$ & Mean & s.d. & Range & Items & Group-level reliability & Cronbach's alpha \\
\hline \multicolumn{9}{|c|}{ General mobility } \\
\hline \multirow[t]{3}{*}{ Child } & Full-item bank & 374 & 49.06 & 10.37 & $18.48-63.04$ & 20 & 0.92 & 0.97 \\
\hline & 10-item CAT & 374 & 49.12 & 10.25 & $19.04-62.76$ & 10 & 0.90 & NA \\
\hline & Short form & 374 & 48.83 & 10.02 & $20.69-61.63$ & 10 & 0.88 & 0.95 \\
\hline \multirow[t]{3}{*}{ Parent } & Full-item bank & 319 & 49.94 & 10.99 & $26.94-69.32$ & 18 & 0.94 & 0.97 \\
\hline & 10-item CAT & 318 & 49.90 & 10.95 & $27.17-69.20$ & 10 & 0.94 & NA \\
\hline & Short form & 319 & 49.80 & 10.46 & $28.85-67.99$ & 9 & 0.91 & 0.95 \\
\hline \multicolumn{9}{|l|}{ Ambulation } \\
\hline \multirow[t]{3}{*}{ Child } & Full-item bank & 123 & 50.12 & 13.72 & $11.96-74.69$ & 25 & 0.95 & 0.97 \\
\hline & 10-item CAT & 123 & 50.05 & 13.71 & $12.04-73.88$ & 10 & 0.94 & NA \\
\hline & Short form & 123 & 50.22 & 12.53 & 22.03-72.46 & 11 & 0.91 & 0.95 \\
\hline \multirow[t]{3}{*}{ Parent } & Full-item bank & 116 & 32.98 & 7.21 & $17.30-50.92$ & 25 & 0.96 & 0.97 \\
\hline & 10-item CAT & 116 & 33.03 & 7.19 & $17.30-50.92$ & 10 & 0.95 & NA \\
\hline & Short form & 116 & 33.42 & 6.58 & $21.93-50.77$ & 10 & 0.92 & 0.94 \\
\hline
\end{tabular}

Abbreviations: CAT, computerized adaptive test; NA, not applicable; PEDI-SCI, Pediatric Spinal Cord Injury.

aseparate short forms for paraplegia and tetraplegia were not developed for these domains.

Table 2 Descriptive statistics of the PEDI-SCI daily routine scales

\begin{tabular}{|c|c|c|c|c|c|c|c|c|}
\hline Respondent & Version & $\mathrm{N}$ & Mean & s.d. & Range & Items & Group-level reliability & Cronbach's alpha \\
\hline \multirow[t]{4}{*}{ Child } & Full-item bank & 372 & 52.45 & 11.32 & $-7.44-75.35$ & 192 & 0.94 & $0.99^{a}$ \\
\hline & 10-item CAT & 372 & 52.08 & 10.79 & 6.43-74.40 & 10 & 0.91 & NA \\
\hline & Paraplegia short form & 214 & 54.70 & 4.13 & $29.08-73.53$ & 11 & 0.79 & $0.88^{b}$ \\
\hline & Tetraplegia short form & 156 & 44.42 & 10.01 & $20.58-58.43$ & 12 & 0.76 & 0.94 \\
\hline \multirow[t]{4}{*}{ Parent } & Full-item bank & 318 & 51.36 & 11.15 & $11.56-75.01$ & 185 & 0.96 & $0.99^{c}$ \\
\hline & 10-item CAT & 317 & 51.19 & 10.97 & $10.01-68.77$ & 10 & 0.93 & NA \\
\hline & Paraplegia short form & 177 & 55.52 & 5.99 & $26.83-62.43$ & 12 & 0.89 & $0.91^{b}$ \\
\hline & Tetraplegia short form & 136 & 43.58 & 8.61 & $25.04-58.24$ & 11 & 0.86 & 0.95 \\
\hline
\end{tabular}

Abbreviations: CAT, computerized adaptive test; NA, not applicable; PEDI-SCI, Pediatric Spinal Cord Injury.

aTo calculate Cronbach's alpha, we removed 54 items not completed by all participants because of the use of specific device.

${ }^{\mathrm{b}}$ Removed 1 item skipped by children not using a bowel program.

'Removed 52 items due to use of specific device. 
Table 3 Descriptive statistics of the PEDI-SCI wheeled mobility scales ${ }^{\mathrm{a}}$

\begin{tabular}{|c|c|c|c|c|c|c|c|c|}
\hline Respondent & Version & N & Mean & s.d. & Range & Items & Group-level reliability & Cronbach's alpha \\
\hline \multirow{3}{*}{ Child } & 10-item CAT & 329 & 48.01 & 13.8 & $-32.91-70.23$ & 10 & 0.89 & NA \\
\hline & Tetraplegia MWC ${ }^{\mathrm{C}}$ short form & 97 & 42.94 & 12.23 & $11.26-67.20$ & 8 & 0.73 & 0.88 \\
\hline & Tetraplegia PWC $C^{d}$ short form & 80 & 34.42 & 13.80 & $-28.03-60.88^{e}$ & 10 & 0.85 & 0.82 \\
\hline \multirow[t]{3}{*}{ Parent } & Full-item bank & 277 & 47.72 & 11.5 & 3.64-70.21 & 64 & 0.96 & $0.98^{f}$ \\
\hline & Tetraplegia MWC short form & 80 & 43.86 & 10.63 & $22.34-66.10$ & 10 & 0.90 & 0.95 \\
\hline & Tetraplegia PWC short form & 61 & 35.59 & 11.65 & $3.36-62.69$ & 9 & 0.83 & 0.80 \\
\hline
\end{tabular}

Abbreviations: CAT, computerized adaptive test; NA, not applicable; PEDI-SCI, Pediatric Spinal Cord Injury.

a Separate SFs for children with paraplegia and tetraplegia; for tetraplegia, separate SFs for power and manual wheelchair.

bTo calculate Cronbach's alpha, we removed 26 items not completed by all participants due to use of specific device.

cManual Wheelchair.

dPower Wheelchair.

eScore is lower because one subject responded to all power wheelchair items as 'unable'.

${ }^{\text {fRemoved }} 25$ items because of the use of specific device.
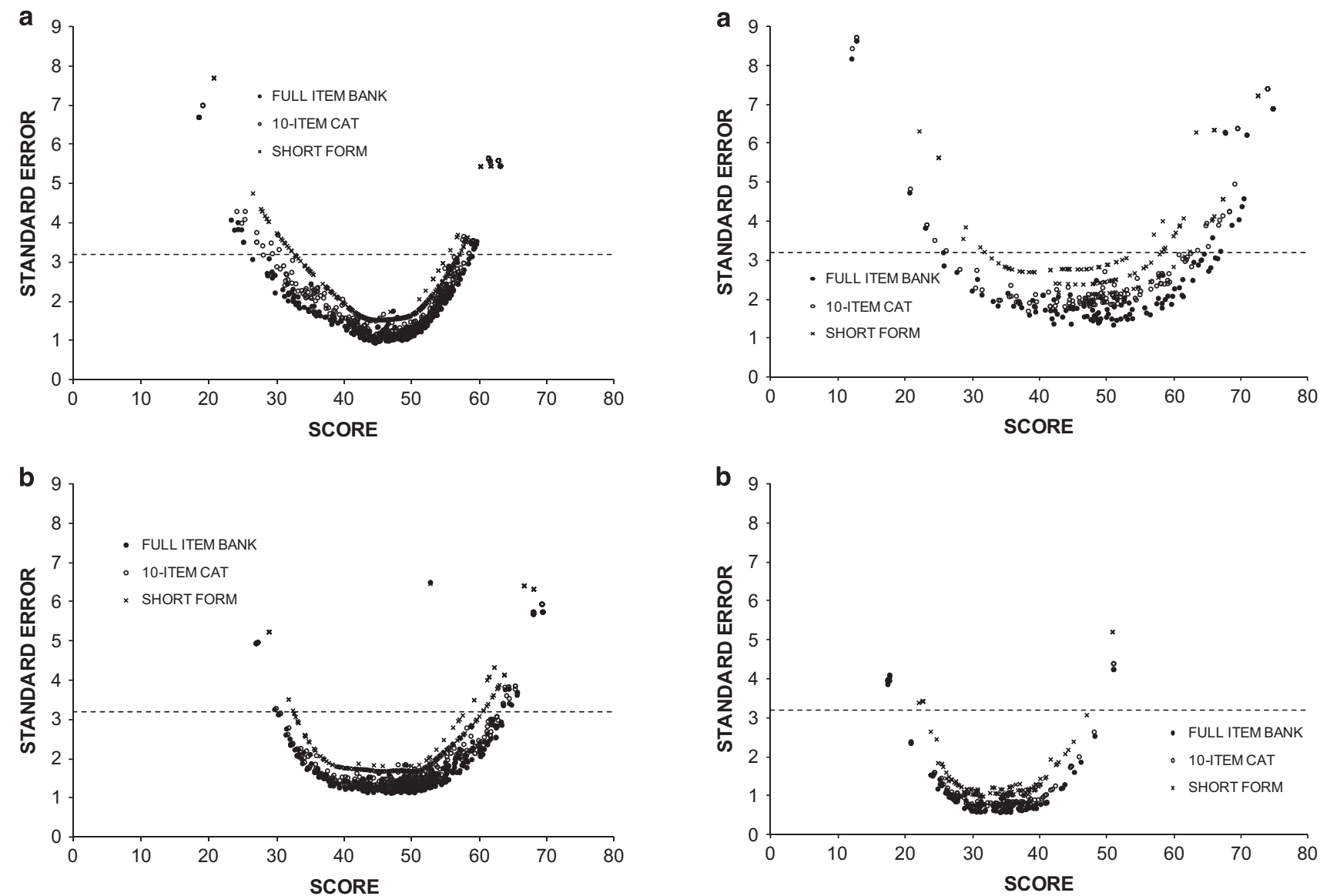

Figure 1 s.e. (dashed line represents reliability $=0.90$ ). (a) General mobility (child reported). (b) General mobility (parent reported).

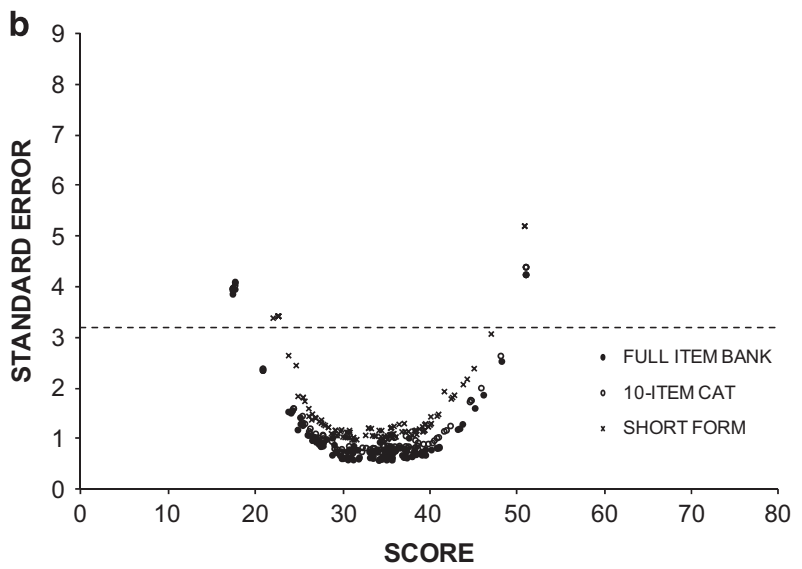

Figure 2 s.e. (dashed line represents reliability=0.90). (a) Ambulation (child reported). (b) Ambulation (parent reported).

parent) defined as follows:

$$
\frac{\sigma_{\theta}^{2}-E\left(S E^{2}\right)}{\sigma_{\theta}^{2}}
$$

where $\mathrm{E}\left(\right.$ s.e. $\left.^{2}\right)$ is the mean of estimated score s.e.'s in each group, and $\sigma_{\theta}^{2}$ is the variance of the estimated score for child and parent respondents. We also calculated Cronbach's alpha for the full-item bank and SF items (but not for 10-item CATs as different items were administered). Intraclass correlation coefficients (ICCs) were calculated to determine agreement between the total item bank and 10-item CATs and SFs. We examined precision of score estimates by limiting the score range ( 0 through 80$)$ and calculating the s.e. Finally, we calculated the percent of the sample with the highest (ceiling) and the lowest (floor) score for the full-item banks, 10-item CATs and SFs. 

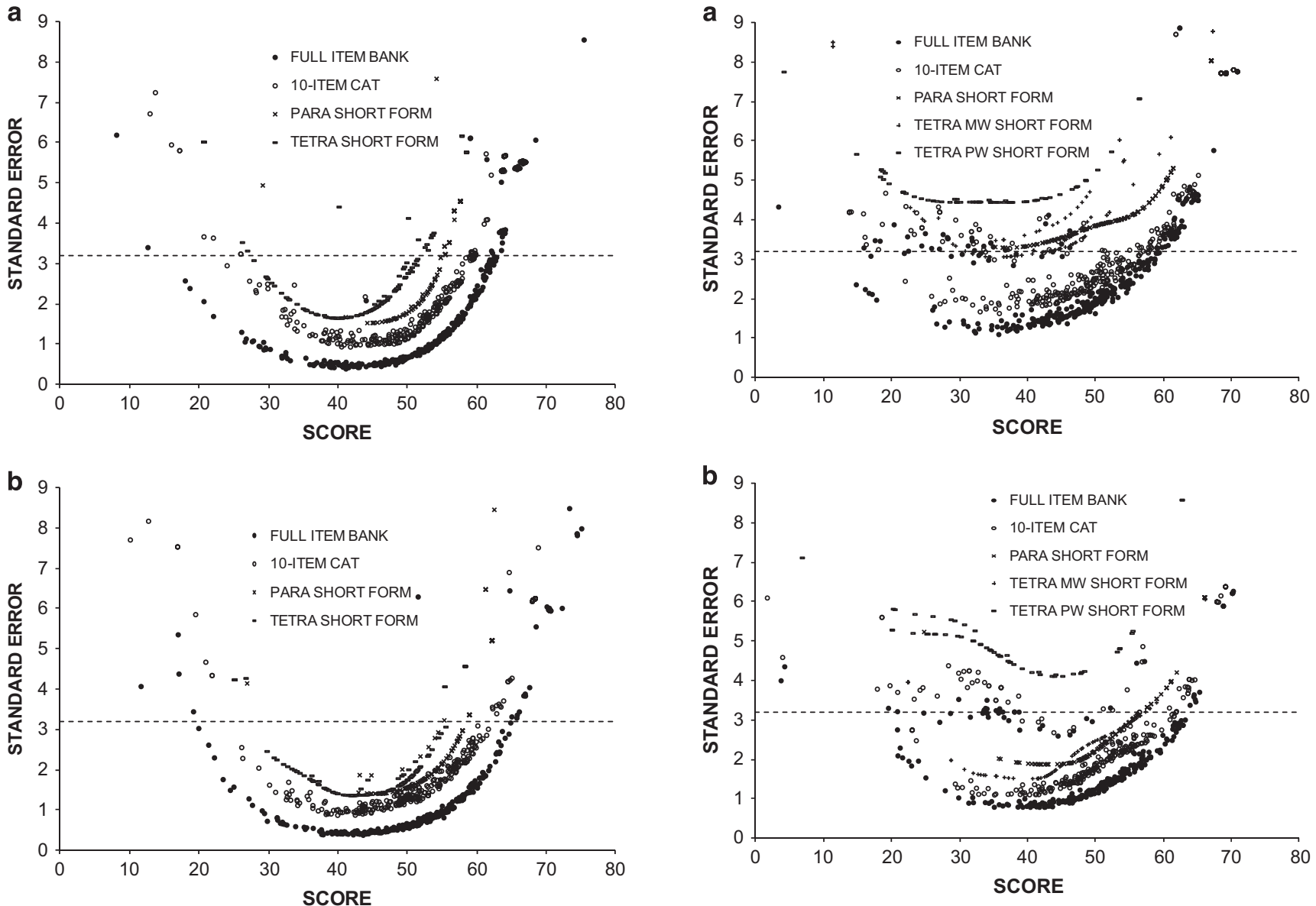

Figure 3 s.e. (dashed line represents reliability=0.90). (a) Daily routine (child reported). (b) Daily routine (parent reported).

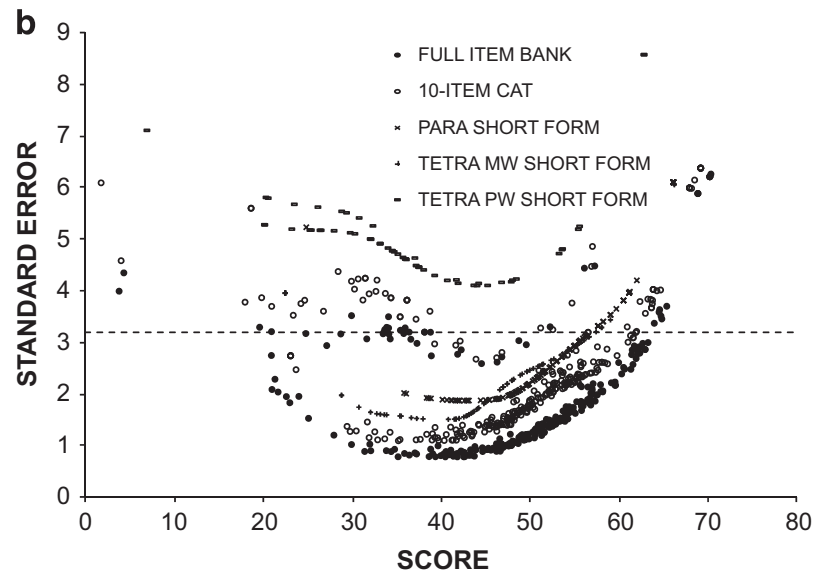

Figure 4 s.e. (dashed line represents reliability $=0.90$ ). (a) Wheeled mobility (child reported). (b) Wheeled mobility (parent reported).

Table 4 PEDI-SCI general mobility and daily routines scales ${ }^{a}$

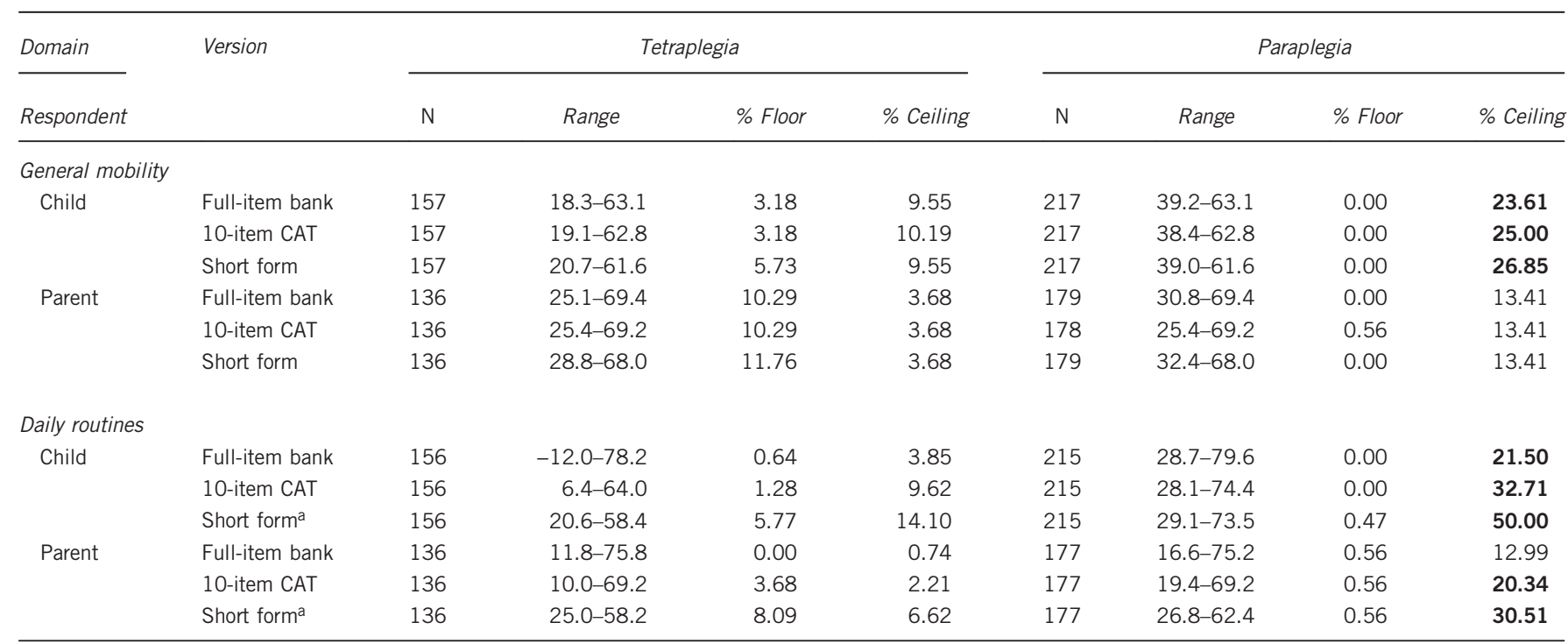

Abbreviations: CAT, computerized adaptive test; PEDI-SCI, Pediatric Spinal Cord Injury.

aSeparate short forms for children with paraplegia and tetraplegia. 


\section{RESULTS}

\section{PEDI-SCI sample}

The mean age (s.d.) of the participants for the child-reported version was 15.5 (s.d. $=3.5$ ) years; most were boys $(55 \%)$ and white $(82 \%)$; $57.6 \%$ had paraplegia; and $54.2 \%$ had complete injuries, as defined by American Spinal Injury Association Impairment Scale (AIS). Mean age (s.d.) for the parent-reported version was 13.6 (s.d. $=4.5$ ) years; most were boys (55\%) and white (82\%); 56\% had paraplegia; and 52\% had complete injuries (AIS). Details are reported elsewhere. ${ }^{7}$

\section{PEDI-SCI item banks}

The PEDI-SCI AM item banks include four domains: general mobility (19 child items and 18 parent items), daily routines (192 child items and 185 parent items), wheeled mobility (62 child items and 64 parent items) and ambulation ( 25 child items and 25 parent items; see Supplementary Material $^{8}$ for complete list of items). Core items $(N=178)$ were administered to all participants; supplemental items $(N=229)$ were administered based on responses to screener questions (for example, age-specific items, bowel and bladder program, and ambulation status). ${ }^{8}$

\section{Descriptive statistics}

Tables 1-3 present the range, mean values and s.d.'s for the full-item banks, 10-item CATs and SFs. Scores were calculated using the $T$ metric $($ mean $=50$; s.d. $=10)$. Mean values were near 50 with some exceptions. For ambulation, parent respondents were below the mean for CAT (33.0) and SF (33.4) administrations. Where separate SFs were developed for different levels of lesion, lower mean scores were noted for tetraplegic-specific SFs, as expected (child and parent daily

Table 5 PEDI-SCI ambulation scales

\begin{tabular}{llccc}
\hline \multirow{2}{*}{ Respondent } & Version & \multicolumn{3}{c}{ Paraplegia and tetraplegia } \\
\cline { 3 - 5 } & & $\mathrm{N}$ & \% Floor & \% Ceiling \\
\hline \multirow{2}{*}{ Child } & Full-item bank & 123 & 2.44 & 7.32 \\
& 10-item CAT & 123 & 2.44 & 8.94 \\
& Short form & 123 & 5.69 & 12.2 \\
Parent & Full-item bank & 116 & 6.03 & 2.59 \\
& 10-item CAT & 116 & 6.03 & 2.59 \\
& Short form & 116 & 10.34 & 2.59 \\
\hline
\end{tabular}

Abbreviations: CAT, computerized adaptive test; PEDI-SCI, Pediatric Spinal Cord Injury. routines tetraplegia SFs $=44.4$ and 43.6 , respectively; child and parent power wheeled mobility tetraplegia $\mathrm{SFs}=34.4$ and 35.6, respectively; and child and parent manual wheeled mobility tetraplegia $\mathrm{SFs}=42.9$ and 43.9, respectively). Higher mean scores were noted for paraplegia-specific SFs (child and parent daily routines paraplegia $\mathrm{SFs}=54.7$ and 55.5, respectively; and child wheeled mobility paraplegia $\mathrm{SF}=54.0$ ).

Group-level reliability (0.73-0.96) was moderate to high for all domains and versions (full-item banks, 10-item CATs and SFs). Internal consistency values for the full-item banks and SFs were also acceptable (Cronbach's alpha $=0.77-0.99$ ). For general mobility and ambulation item banks (child and parent versions), agreement was high for the CATs and SFs (ICC range $=0.97-0.99$ ). For the daily routines and wheeled mobility item banks (child and parent versions), agreement was high for the CATs (ICC $=0.97-0.99$ ); however, for daily routines, agreement was lower for paraplegia-specific SFs for children $(\mathrm{ICC}=0.72)$ and parents $(\mathrm{ICC}=0.83)$ compared with tetraplegia-specific SFs for children $(\mathrm{ICC}=0.93)$ and parents (ICC $=0.95)$. For wheeled mobility SFs, agreement was strong (ICC range $=0.90-0.99$ ).

\section{Score estimate precision}

Figures 1-4 display the s.e. observed for each domain and version. The dashed line represents reliability at 0.90 ; points below the line indicate the score reliability $>0.9$ and above the line score reliability $<0.9$. The general mobility (Figure 1) and ambulation (Figure 2) plots demonstrate excellent precision for low mid-range score estimates that varies somewhat by version (full-item banks $>10$-item CATs $>$ SFs). For general mobility, the precision for parent and child respondents is similar. For ambulation, precision is better for parent respondents, but it should be noted that the sample size is smaller as fewer children were ambulatory. Results for daily routines (Figure 3) should be viewed with caution because of the problems noted with ceiling effects; however, a similar pattern is noted. Finally, for wheeled mobility (Figure 4), precision estimates vary by mode of administration with high s.e.'s for tetraplegia SFs and 10-item CATs for both child and parent respondents; however, these instruments were completed by subsets of the sample (for example, only manual wheelchair users).

\section{Ceiling and floor effects}

Tables 4-6 present floor and ceiling effects for the PEDI-SCI banks, 10 -item CATs and SFs. Floor effects are acceptable $(<15 \%)$ across all domains, respondent types and administration versions for children

Table 6 PEDI-SCI wheeled mobility scales ${ }^{\mathrm{a}}$

\begin{tabular}{|c|c|c|c|c|c|c|c|c|c|c|}
\hline \multirow[t]{3}{*}{ Respondent } & \multirow[t]{3}{*}{ Version } & \multicolumn{6}{|c|}{ Tetraplegia } & \multicolumn{3}{|c|}{ Paraplegia } \\
\hline & & \multicolumn{3}{|c|}{ Manual } & \multicolumn{3}{|c|}{ Power } & \multirow[t]{2}{*}{$\mathrm{N}$} & \multirow[t]{2}{*}{$\%$ Floor } & \multirow[t]{2}{*}{$\%$ Ceiling } \\
\hline & & $\mathrm{N}$ & $\%$ Floor & $\%$ Ceiling & $\mathrm{N}$ & $\%$ Floor & $\%$ Ceiling & & & \\
\hline \multirow[t]{3}{*}{ Child } & Full-item bank & 97 & 0.00 & 5.15 & 80 & 1.25 & 6.25 & 189 & 0.00 & 7.41 \\
\hline & 10-item CAT & 98 & 0.71 & 5.00 & 80 & 0.71 & 5.00 & 191 & 0.00 & 9.52 \\
\hline & Short forma & 98 & 4.12 & 8.25 & 80 & 1.25 & 6.25 & 191 & 0.00 & 15.43 \\
\hline \multirow[t]{3}{*}{ Parent } & Full-item bank & 80 & 2.27 & 4.55 & 61 & 0.00 & 0.00 & 153 & 1.19 & 4.76 \\
\hline & 10-item CAT & 80 & 2.27 & 4.55 & 61 & 2.27 & 4.55 & 153 & 0.00 & 9.52 \\
\hline & Short form ${ }^{a}$ & 80 & 4.55 & 4.55 & 61 & 0.00 & 0.00 & 153 & 1.20 & 8.43 \\
\hline
\end{tabular}

Abbreviations: CAT, computerized adaptive test; PEDI-SCI, Pediatric Spinal Cord Injury.

${ }^{a}$ Separate short forms for children with paraplegia and tetraplegia. 
with paraplegia and tetraplegia (range $=0-10.29 \%$ ). Ceiling effects for children with tetraplegia are acceptable $(<15 \%)$ across all domains, respondent types and administration versions. For children with paraplegia, ceiling effects for the general mobility domain are acceptable $(<15 \%)$ for parent respondents but high for child respondents across all versions (23.61-26.85\%). A significant problem with ceiling effects is noted for daily routines in children with paraplegia; ceiling effects are unacceptable, except for the parent daily routine full-item bank (13.0\%). For wheeled mobility, ceiling effects are acceptable for parent and child respondents for all versions, except the child wheeled mobility SF (15.43\%).

\section{DISCUSSION}

The PEDI-SCI AM is the first activity limitation measure developed specifically for youth with SCI. Initial examination of the PEDI-SCI AM demonstrated strong reliability and internal consistency for full-item banks, 10-item CATs and SFs. Simulated 10-item CAT and most SF scores provide precise estimates and show strong agreement with the full-item bank, indicating that CAT programs select appropriate items and SF items that adequately represent the full-item banks. The exception is the daily routine SF for children with paraplegia where the lower agreement with the item bank may limit item selection due to high ceiling effects.

Analysis of floor and ceiling effects indicated that the range of difficulty represented in the ambulation and wheelchair items was appropriate for youth with paraplegia and tetraplegia. Floors effects are minimal $(<15 \%)$ across all domains and respondent types and the full-item banks, 10-item CATs and SFs, indicating that all PEDI-SCI AM measures had sufficient content range for all children, regardless of their level of lesion.

The PEDI-SCI AM measures had the necessary content range at the upper end of the scale for youth with tetraplegia, as evidenced by small ceiling effects. Likewise, for youth with paraplegia, ceiling effects for the child and parent versions of the mobility and ambulation SFs were acceptable. However, for children with paraplegia, ceiling effects for the child and parent daily routines and the child general mobility SFs were unacceptably high for all versions and may underestimate a child's full ability in these domains, especially if the child was functioning at a relatively high level.

The ability to improve the psychometric properties of a measure by adding and calibrating new items (referred to as item bank replenishment) is a significant advantage of Item Response Theorybased measures. The next step in refinement of the PEDI-SCI AM involves replenishment of the daily routines and general mobility item banks. Replenishment can enhance item banks without altering the underlying scale, and we have successfully replenished other item banks. ${ }^{12}$ We will develop new items to assess the upper range of abilities and calibrate these items in a sample of youth with SCI, and these new items will be added to the existing item banks and SFs.

Given the void in robust outcome instruments for pediatric SCI, the PEDI-SCI AM is an important contribution that can elevate pediatric SCI practices. PEDI-SCI AM is the first measure with items specifically developed for youth with SCI that uses sophisticated measurement approach. It can be administered as a SF or CAT; although the PEDISCI AM CATs performed better than the SFs, some SFs were comparable to the simulated 10 -item CAT, providing evidence that the SFs can be an acceptable alternative to CAT administration. Young children are capable of self-reporting on their health conditions; however, discordance between child and parent reports of healthrelated quality of life outcomes has been established in clinical samples and typically developing children. ${ }^{13,14}$ It is important to assess outcomes from both parent and child perspectives, ${ }^{14}$ and the PEDI-SCI AM offers parent and child versions. These versions are based on different metrics, and scores cannot be directly compared, but the ability to administer the measure to parent and child respondents provides clinicians and researchers with different options based on the specific situation and purpose of the assessment. Finally, PEDI-SCI AM can be used with its companion PEDI-SCI participation measure, ${ }^{4}$ and, as item banks are linked to the SCI-FI, an Item Response Theory-based measure developed for the adult population, function can be tracked across the lifespan. ${ }^{8}$ The next step is to validate these measures in a new sample and compare the responsiveness of the PEDI-SCI AM CATs and SFs to generic measures.

There are several limitations to this work: the sample drawn from three Shriners Hospitals for Children in the United States may not be representative; and the sample is relatively small but acceptable given the overall number of children with SCI (estimated number of new injuries is 1500 per year). ${ }^{15}$

\section{CONCLUSION}

The PEDI-SCI AM uses contemporary measurement approaches to create new instruments for pediatric SCI research and clinical practice. For the first time, activity outcomes can be assessed with a measure developed specifically for youth with SCI. The ability to administer any or all of the four domains and select child and/or parent as respondent(s) provides a comprehensive measure that can be customized for multiple purposes, and the ability to use PEDI-SCI AM CAT or SF versions provides administrative options for different settings.

\section{DATA ARCHIVING}

There were no data to deposit.

\section{CONFLICT OF INTEREST}

The authors declare no conflict of interest.

\section{ACKNOWLEDGEMENTS}

The study was funded by the Shriners Hospitals for Children Research Grant 79142 (MJM, PI) and the National Center for Medical Rehabilitation Research-Medical Rehabilitation Research Infrastructure Network 5R24HD065688-04 (AMJ, PI). We acknowledge the following individuals who participated in the pediatric spinal cord injury expert panel that selected short form items: Pip Campbell, PhD, OTRIL; Michelle Gorenberg, OTD; Stephen Kern, PhD, OTR LL; Amanda Horley, MSOT; Therese Johnston, PT, PhD; Ralph Marino, MD; Heather Russell, PhD; and Mary Schmidt-Read, DPT.

1 Msall ME, DiGaudio K, Rogers BT, LaForest S, Catanzaro NL, Campbell J et al. The Functional Independence Measure for Children (WeeFIM). Conceptual basis and pilot use in children with developmental disabilities. Clin Pediatr (Phila) 1994; 33: 421-430.

2 Varni J, Seid M, Rode C. The PedsQL: measurement model for the pediatric quality of life inventory. Med Care 1999; 37: 126-139.

3 Garcia R, Gaebler-Spira D, Sisung C, Heinemann A. Functional improvement after pediatric spinal cord injury. Am J Phys Med Rehabil 2002; 81: 458-463.

4 Calhoun C, Riley A, Haley S, Mulcahey M. Item development for a new measure of activity performance and participation among children with spinal cord injury. Int $J$ Pediatric 2009, 854-904.

5 Bent L, Mulcahey M, Kelly E, Calhoun C, Tian F, Ni P et al. Validity of computer adaptive tests of daily routines for youth with spinal cord injury. Top Spinal Cord Inj Rehabil 2013; 19. 104-113.

6 Mulcahey M, Calhoun C, Riley A, Haley S. Children's reports of activity and participation after sustaining spinal cord injury: a cognitive interviewing study. Dev Neurorehabil 2009; 12: 191-200.

7 Mulcahey M, Calhoun C, Tian F, Ni P, Vogel L, Haley S. Evaluation of newly developed item banks for child-reported outcomes of participation following spinal cord injury. Spinal Cord 2012; 50: 915-919. 
8 Tian F, Ni P, Mulcahey M, Hambleton R, Tulsky D, Haley S et al. Tracking functional status across the spinal cord injury lifespan: linking pediatric and adult patient-reported outcome scores. Arch Phys Med Rehabil 2014; 95 2078-2085.

9 Jette A, Haley S. Contemporary measurement techniques for rehabilitation outcome assessment. J Rehabil Med 2005; 37: 339-345.

10 Warm T. Weighted likelihood estimation of ability in item response theory. Psychometrika 1989; 54: 427-450.

11 Heinemann A, Dijkers M, Ni P, Tulsky D, Jette A. Measurement properties of the Spinal Cord Injury-Functional Index (SCI-FI) short forms. Arch Phys Med Rehabil 2014; 95 1289-1297.
12 Haley S, Ni P, Jette A, Tao W, Moed R, Meyers D et al. Replenishing a computerized adaptive test of patient-reported daily activity functioning. Qual Life Res 2009; 18 : 461-471.

13 Theunissen N, Vogels T, Koopman H, Verrips G, Zwinderman K, Verloove-Vanhorick S et al. The proxy problem: child report versus parent report in health-related quality of life research. Qual Life Res 1998; 7: 387-397.

14 Vetter T, Bridgewater C, McGwin G. An observational study of patient versus parental perceptions of health-related quality of life in children and adolescents with a chronic pain condition: who should the clinician believe? Health Qual Life Outcomes 2012; 10: 85.

15 Chen Y, Tang Y, Vogel L, DeVivo M. Epidemiology of pediatric SCl: causes of spinal cord injury. Top Spinal Cord Inj Rehabil 2013; 19: 1-8.

Supplementary Information accompanies this paper on the Spinal Cord website (http://www.nature.com/sc) 